\title{
Synthesis and Proteasome Inhibition of Glycyrrhetinic Acid Derivatives
}

\author{
Li Huang ${ }^{1}$, Donglei $\mathrm{Yu}^{2}$, Phong Ho ${ }^{1}$, Keduo Qian ${ }^{2}$, Kuo-Hsiung Lee ${ }^{2}$, and Chin-Ho Chen ${ }^{{ }^{*}}$ \\ 1Department of Surgery, Duke University Medical Center, Durham, North Carolina 27710, USA \\ 2Natural Products Research Laboratories, School of Pharmacy, University of North Carolina, Chapel Hill, \\ North Carolina 27599, USA
}

\begin{abstract}
This study discovered that glycyrrhetinic acid inhibited the human 20S proteasome at $22.3 \mu \mathrm{M}$. Esterification of the C-3 hydroxyl group on glycyrrhetinic acid with various carboxylic acid reagents yielded a series of analogs with marked improved potency. Among the derivatives, glycyrrhetinic acid 3-O-isophthalate (17) was the most potent compound with $\mathrm{IC}_{50}$ of $0.22 \mu \mathrm{M}$, which was approximately 100 -fold more potent than glycyrrhetinic acid.
\end{abstract}

\section{Keywords}

Glycyrrhetinic acid; proteasome inhibitor; triterpene

\section{Introduction}

$18 \beta$-glycyrrhetinic acid (GLA) is the aglycone of glycyrrhizic acid, a major component found in licorice root that has been widely used in traditional medicines, especially in Asia (1). The known pharmacological activities of GLA and glycyrrhizic acid include anti-inflammation, anti-ulcer, anti-allergenic, and anti-viral. The GLA derivative, carbenoxolone, has been used in Europe as a licensed medicine for the treatment of esophageal ulceration and inflammation (2). GLA was shown to have multiple biological activities, including inhibition of 11- $\beta$ hydroxysteroid dehydrogenase and induction of mitochondrial permeability transition (3-5). How these biological activities contribute to the pharmacological effect of GLA remains to be determined.

The proteasome-ubiquitin pathway is known for breaking down proteins to remove the misfolded or damaged proteins in eukaryotic cells $(6,7)$. In the past decade, increasing evidence has indicated that the proteasome-ubiquitin system plays an important role in many cellular functions such as cell stress response, cell cycle regulation, cellular differentiation, and antigenic peptide generation. Therefore, targeting the proteasome-ubiquitin pathway has been considered a novel strategy for the treatment of various disorders such as neurodegenerative diseases, cancers, and inflammatory diseases (8).

*Corresponding author. Chin-Ho Chen, Department of Surgery, Box 2926, Duke University Medical Center, Durham, NC 27710-2926, U.S.A. Tel.: 919-684-3819; Fax: 919-684-3878; E-mail: chc@ duke.edu.

Publisher's Disclaimer: This is a PDF file of an unedited manuscript that has been accepted for publication. As a service to our customers we are providing this early version of the manuscript. The manuscript will undergo copyediting, typesetting, and review of the resulting proof before it is published in its final citable form. Please note that during the production process errors may be discovered which could affect the content, and all legal disclaimers that apply to the journal pertain. 
The core structure of the proteasome is a barrel-shaped $20 \mathrm{~S}$ complex that has four stacked rings each with 7 subunits (8). The two outer rings and two inner rings are named $\alpha$ rings and $\beta$ rings, respectively. The $20 \mathrm{~S}$ proteasome is activated when the regulatory protein complex PA700 or PA28 binds to the $\alpha$ ring that opens the channel in the 20S proteasome and allows the ubiquitintagged proteins to access the proteolytic sites located inside the chamber. Within the $\beta$ rings, there are multiple proteolytic sites including two chymotrypsin-like (ChT-L), two trypsin-like (T-L), and two caspase-like (CA-L) proteolytic sites (10). The proteasome inhibitor PS341 (bortezomib) was successfully developed into an anti-cancer drug for the treatment of multiple myeloma (11). Differential inhibition of the three catalytic activities by bortezomib is believed to be critical for clinical benefit in disease treatment (12). Recently, we reported that betulinic acid (BA) and its derivatives could regulate the proteasome activities (13). BA acted as an activator of the $20 \mathrm{~S}$ proteasome complex. On the other hand, some of the BA derivatives were shown to be inhibitors of the $20 \mathrm{~S}$ proteasome.

\section{Results and discussion}

In this study, we continued our effort of searching for new compounds that possess proteasomal regulatory activity. Several triterpenes with structures similar to BA were tested for their potentials in regulating the proteasome, including glycyrrhetinic acid (GLA, 1), oleanolic acid (OA), ursolic acid (UA), and moronic acid (MA) as shown in Figure 1. The compounds were initially examined for their effect on the ChT-L activity of the proteasome. The ChT-L activity is the most critical enzymatic process in the proteasome. Genetic studies suggested that impaired proteasome ChT-L activity caused by mutations resulted in strong reduction in the degradation of proteasomal substrates (14-17), whereas impaired T-L or CA-L activity of proteasomes did not cause such a detrimental effect. Our current study indicated that among these five triterpenes, GLA is the only one that inhibited the ChT-L activity of the proteasome. GLA reduced the proteasome activity by $50 \%$ at $22.3 \mu \mathrm{M}$. MA did not inhibit or activate proteasome activity, possibly due to the lack of a C-3 hydroxyl group. On the contrary, BA, $\mathrm{UA}$, and OA all activated the ChT-L activity of the proteasome (data not shown).

\section{Synthesis and characterization of GLA derivatives}

Our previous study indicated that BA derivatives with side chain modifications at the C-3 position transformed BA into proteasome inhibitors. In an attempt to increase the potency of GLA's inhibitory effect on proteasome, a series of GLA derivatives functionalized with a variety of C-3 side chains were synthesized. The synthesis of GLA C-3 ester derivatives was accomplished by treating GLA with corresponding carboxylic acid reagents under general esterification conditions. Compounds 2-7, 9 and 11 were obtained with corresponding anhydrides in the presence of DMAP and pyridine using microwave assisted heating. The rest of the compounds $(\mathbf{8}, 10$ and 12-18) were synthesized by coupling the corresponding carboxylic acid reagents in the presence of $\mathrm{EDC} \cdot \mathrm{HCl} / \mathrm{DMAP}$ or DCC/DMAP under microwave irradiation. Compounds 19-21 were modified at the C-30 position with amide moieties with or without additional modification on their $\mathrm{C}-3$ position. The synthesis of these compounds has been previously reported (18).

The structures of four of the most potent GLA derivatives, $\mathbf{8}, \mathbf{1 2}, \mathbf{1 7}$, and $\mathbf{1 8}$, were further confirmed with ${ }^{13} \mathrm{C}$ NMR studies (Table 1). The spectra have shown all 30 carbons of GLA triterpene skeletons with unambiguous assignment for 7 of them based on comparison of data reported before for GLA (19). The carbon signals of C-3 ester side chain were also observed except for the three overlapping signals in compound 18. In summary, the spectra showed no or very small difference of chemical shift (all within $0.2 \mathrm{ppm}$ except for the C-3) among the four compounds on the triterpene skeleton region due to its structural rigidity and stability. The 
C-3 chemical shifts showed more variety among these GLA esters and were found to be more than 2 ppm downfield than that in GLA.

\section{Inhibition of the proteasome by GLA derivatives}

The synthesized compounds were assayed at various concentrations in order to determine their potency against the ChT-L activity (13). The known proteasome inhibitors Ac-Leu-Leu-MetCHO (LLM-F) and lactacystin were included in the assay as controls. The results indicated that C-3 esterification of GLA increased the potency of proteasome inhibition by 3 to 100 -fold when compared with unmodified GLA (Table 2). It appeared that compounds with aromatic $\mathrm{C}-3$ side chains as seen in 13-18 were, in general, more potent $\left(\mathrm{IC}_{50}=0.22-2.27 \mu \mathrm{M}\right)$ than compounds without the aromatic side chain except for compounds 8 and 12. Compound 8 possessed an unbranched side chain similar to compounds 4 and $\mathbf{6}$. The length of the side chains of these compounds was positively correlated with the potency of the compounds against the proteasome. For example, the C-3 side chains of compounds 4, 6 , and 8 increased from $\mathrm{C}_{4}$, $\mathrm{C}_{5}$, to $\mathrm{C}_{6}$, respectively, and this correlated with the improved potency of these compounds. The $50 \%$ inhibitory concentrations $\left(\mathrm{IC}_{50}\right.$ ) of compounds $\mathbf{4 , 6}$, and $\mathbf{8}$ were $2.63,1.39$, and 0.29 $\mu \mathrm{M}$, respectively. Compound 12, containing an unsaturated side chain, also exhibited potent inhibitory activity with $\mathrm{IC}_{50}$ equal to $0.35 \mu \mathrm{M}$.

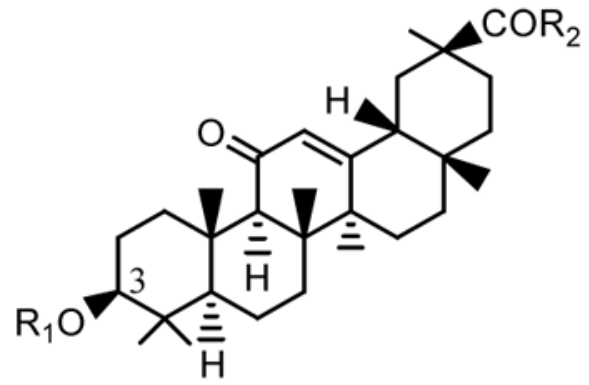

\section{8 - $\beta$-glycyrrhetinic acid derivatives}

The free carboxylic acid moiety in the $\mathrm{C}-3$ side chain was not required for proteasome inhibition as shown with compounds $\mathbf{1 1}, \mathbf{1 3}$, and $\mathbf{1 4}$, which exhibited moderate potency against proteasome. However, a free carboxylic acid moiety appeared to enhance the potency of the compound. For example, the carboxylic acid on compounds 17 and 18 might be responsible for the 10-fold increase in the anti-proteasomal activity when compared with compound $\mathbf{1 3}$.

Additional side chain modification at C-30 significantly decreased the potency of the C-3 derivatives of GLA. The $\mathrm{IC}_{50}$ of the $\mathrm{C}-3$ derivative, 2 , was $0.87 \mu \mathrm{M}$ against the ChT-L activity of the proteasome. Addition of a C-30 side chain resulted in compound 21 that inhibited the proteasomal activity at $3.09 \mu \mathrm{M}$.

One of the possible reasons that bortezomib can selectively inhibit cancer cells could be due to its preferential inhibitory activity against the ChT-L activity of the proteasome $(12,20)$. To determine whether GLA derivatives can also preferentially inhibit the ChT-L activity of the proteasome, GLA and three of the most potent compounds, $\mathbf{8}, \mathbf{1 2}$, and 17, were tested for their effects on the T-L and CA-L activities of the proteasome. These two proteolytic activities were analyzed using the assays previously described (13). In general, the GLA derivatives were at least 10-fold less potent against the CA-L or T-L activity when compared with their anti-ChT$\mathrm{L}$ activity of the proteasome (Table 2). These results suggested that all of the three tested compounds exhibited preferential inhibitory activity against the ChT-L activity of the proteasome. 
To determine whether GLA derivatives could inhibit the proteasome in the cells, 17, the most potent compound of the series, was tested in a cell-based proteasome assay previously described (13). To determine the effect of $\mathbf{1 7}$ on the ChT-L activity of the proteasome in living cells, MT4 cells were treated with $\mathbf{1 7}$ or the known proteasome inhibitors LLM-F and lactacystin. The ChT-L activity of the proteasome in the cells was analyzed using a Promega cell-based proteasome assay kit and protocol. In this study, compound $\mathbf{1 7}$ inhibited the ChT$\mathrm{L}$ activity of the proteasome by $50 \%$ at approximately $0.25 \mu \mathrm{M}$ (Figure 2). The inhibitory activity of $\mathbf{1 7}$ in the cell-based assay was comparable to the purified human 20S proteasome assay. On the other hand, lactacystin was approximately one $\log _{10}$ more potent in the cellbased assay when compared with that in the purified 20S proteasome assay. It is possible that the metabolites of lactacystin in the cells are responsible for the increased potency (21)

\section{Inhibition of IKB degradation in MT4 cells}

Proteasome degradation of $\mathrm{I} \kappa \mathrm{B} \alpha$ is required for $\mathrm{NF} \kappa \mathrm{B}$ activation by tumor necrosis factor alpha

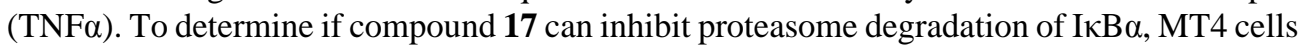
were treated with $17(1.6 \mu \mathrm{M})$ for 3 hours before treating with TNF $\alpha(10 \mathrm{ng} / \mathrm{ml})$ for 30 minutes.. The cytosolic proteins were analyzed in a $12 \%$ SDS polyacrylamide gel. The proteins in the gel were transferred to a nitrocellulose paper for Western blot analyses. An IкB $\alpha$ monoclonal antibody (Abcam, Cambridge, UK) was used to detect IкB $\alpha$ in the samples. TNF $\alpha$ treatment resulted in a significant reduction in I $\mathrm{KB} \alpha$ level when compared with the control in the absence of TNF $\alpha$ (Figure 3) The TNF $\alpha$-induced proteasome degradation of I $\kappa \mathrm{B} \alpha$ was significantly inhibited in the presence of $\mathbf{1 7}$. In contrast, $\beta$-actin level in the cells was not affected by $\mathbf{1 7}$. The results suggested that $\mathbf{1 7}$ specifically inhibited the proteasome in the cells.

\section{Conclusion}

In summary, this study shows that GLA is a proteasome inhibitor, and the potency of this inhibitory activity could be markedly improved through chemical modification at the C-3 position of the compound. GLA is a natural product and has abundant natural sources. It can be obtained from the hydrolysis of the glycoside glycyrrhizic acid (GL). GL from licorice has been used as a food sweetener and in clinical treatment of $\mathrm{HBV}$, where it appeared to have low toxicity and side effects (22). GLA was found to be the major component in serum after ingestion of GL (23). Therefore, development of GLA derivatives as proteasome inhibitors might have the potential to provide new therapeutics for diseases such as cancers and inflammatory diseases.

\section{Experimental}

\section{General experimental procedures}

The Biotage Initiator 2.0 was used for microwave assisted synthesis. All melting points were determined with a Fisher-Johns melting point apparatus without correction. Positive and negative FABHRMS were recorded on a Joel SX-102 spectrometer. ${ }^{1} \mathrm{H}(300 \mathrm{MHz})$ and ${ }^{13} \mathrm{C}$ (125 MHz) NMR spectra were measured on a Varian Mercury 300 spectrometer and Varian Inova 500 spectrometer, respectively. Other than as noted, all samples were dissolved in $\mathrm{CDCl}_{3}$ with TMS as internal standard. Silica gel chromatography was carried out on a Biotage Horizon Flash chromatograph system with pre-packed Si gel column. HPLC was performed on a Varian ProStar solvent delivery and PDA detector with Agilent Zorbax ODS or C-8 columns $(4.6 \mathrm{~mm} \times 25 \mathrm{~cm}$ and $9.4 \mathrm{~mm} \times 25 \mathrm{~cm}$ for analytical and semi preparative scales, respectively). The HPLC profile of each new compound was obtained under two solvent systems (Table 3 ). 


\section{Procedure A for coupling at the C-3 hydroxyl group}

A mixture of GLA, anhydride (5 10 eq.), and DMAP (1 eq.) in pyridine (anhydrous) was heated for 0.5 to $2 \mathrm{hr}$ at 100 to $130^{\circ} \mathrm{C}$ in microwave synthesizer. The mixture was then concentrated under vacuum, re-dissolved in $\mathrm{MeOH}$, and purified with Si-gel chromatography or reverse phase HPLC. The yields ranged from 30 to $50 \%$.

\section{Procedure B for coupling at the C-3 hydroxyl group}

A solution of di-carboxylic acid (10 15 eq.), DCC (5 10 eq.), and DMAP (1 eq.) in $\mathrm{CH}_{2} \mathrm{Cl}_{2}$ (anhydrous) was added to GLA in pyridine and heated for 0.5 to $2 \mathrm{hr}$ at 100 to $130^{\circ} \mathrm{C}$ in microwave synthesizer. The yields ranged from 20 to $40 \%$.

\section{3ß-O-(2',3'-Dimethylsuccinyl)-18- $\beta$-glycyrrhetinic acid (3)}

Positive FABMS $m / z 599.5(\mathrm{M}+\mathrm{H})^{+}$; HR-FABMS calcd for $\mathrm{C}_{36} \mathrm{H}_{53} \mathrm{O}_{7} 597.3791$, found 597.3783. ${ }^{1} \mathrm{H}$ NMR $\delta 5.69(1 \mathrm{H}, \mathrm{s}, \mathrm{H}-12), 4.52(1 \mathrm{H}, \mathrm{dd}, J=4.5 \mathrm{~Hz}, J=11.0 \mathrm{~Hz}, \mathrm{H}-3), 2.74-$ $2.89\left(3 \mathrm{H}, \mathrm{m}, \mathrm{H}_{2 \alpha}\right.$ and $\left.2 \times \mathrm{CH}-\mathrm{COO}\right), 1.36\left(3 \mathrm{H}\right.$, s. $\left.\mathrm{CH}_{3}-29\right), 1.24-1.26\left(6 \mathrm{H}, \mathrm{m}, 2 \times \mathrm{C}_{3}-\mathrm{CHCOO}\right)$, $1.21\left(3 \mathrm{H}\right.$, s. $\left.\mathrm{CH}_{3}-25\right), 1.16\left(3 \mathrm{H}\right.$, s. $\left.\mathrm{CH}_{3}-23\right), 1.12\left(3 \mathrm{H}\right.$, s. $\left.\mathrm{CH}_{3}-24\right), 0.87,0.88$ (each $3 \mathrm{H}$, each s, $\mathrm{CH}_{3}-26$ and $\left.\mathrm{CH}_{3}-27\right), 0.83\left(3 \mathrm{H}, \mathrm{CH}_{3}-28\right)$.

\section{$3 \beta-0-S u c c i n y l-18-\beta$-glycyrrhetinic acid (4)}

Positive FABMS $m / z 571.4(\mathrm{M}+\mathrm{H})^{+}$; HR-FABMS calcd for $\mathrm{C}_{34} \mathrm{H}_{49} \mathrm{O}_{7} 569.3478$, found 569.3465. \%. ${ }^{1} \mathrm{H}$ NMR $\left(\mathrm{CDCl}_{3} / \mathrm{Py}-\mathrm{d}_{5}\right) \delta 5.69(1 \mathrm{H}, \mathrm{s}, \mathrm{H}-12), 4.52(1 \mathrm{H}, \mathrm{dd}, J=5.7 \mathrm{~Hz}, J=11.4$ $\mathrm{Hz}, \mathrm{H}-3), 2.75\left(1 \mathrm{H}, \mathrm{d}, J=14.4 \mathrm{~Hz}, \mathrm{H}_{2 \alpha}\right), 2.63\left(4 \mathrm{H}, \mathrm{s}, 2 \times \mathrm{CH}_{2} \mathrm{CO}\right), 1.33\left(3 \mathrm{H}, \mathrm{s} . \mathrm{CH}_{3}-29\right), 1.17$ $\left(3 \mathrm{H}, \mathrm{s} . \mathrm{CH}_{3}-25\right), 1.10\left(3 \mathrm{H}, \mathrm{s} . \mathrm{CH}_{3}-23\right), 1.06\left(3 \mathrm{H}, \mathrm{s} . \mathrm{CH}_{3}-24\right), 0.83\left(6 \mathrm{H}, \mathrm{s}, \mathrm{CH}_{3}-26\right.$ and $\left.\mathrm{CH}_{3}-27\right), 0.76\left(3 \mathrm{H}, \mathrm{CH}_{3}-28\right)$.

\section{3ß-O-(3',3'-Dimethylglutaryl)-18- $\beta$-glycyrrhetinic acid (5)}

Positive FABMS $m / z 613.5(\mathrm{M}+\mathrm{H})^{+}$; HR-FABMS calcd for $\mathrm{C}_{37} \mathrm{H}_{55} \mathrm{O}_{7} 611.3948$, found 611.3930. ${ }^{1} \mathrm{H}$ NMR $\left(\mathrm{CDCl}_{3} / \mathrm{Py}-\mathrm{d}_{5}\right) \delta 5.58(1 \mathrm{H}, \mathrm{s}, \mathrm{H}-12), 4.48(1 \mathrm{H}, \mathrm{dd}, J=5.2 \mathrm{~Hz}, J=11.0$ $\mathrm{Hz}, \mathrm{H}-3), 2.64\left(1 \mathrm{H}, \mathrm{d}, J=13.3 \mathrm{~Hz}, \mathrm{H}_{2 \alpha}\right), 2.39-2.52\left(4 \mathrm{H}, \mathrm{m}, 2 \times \mathrm{COCH}_{2}\right), 1.29\left(3 \mathrm{H}, \mathrm{s} . \mathrm{CH}_{3}-29\right)$, $1.23\left(3 \mathrm{H}, \mathrm{s} . \mathrm{CH}_{3}-25\right), 1.16$ (3H, s. $\left.\mathrm{CH}_{3}-23\right), 1.14,1.13$ (each $3 \mathrm{H}$, each s, $\left.\left[\mathrm{CH}_{3}\right]_{2} \mathrm{C}\right), 1.08(3 \mathrm{H}$, s. $\left.\mathrm{CH}_{3}-24\right), 0.83,0.84$ (each $3 \mathrm{H}$, each s, $\mathrm{CH}_{3}-26$ and $\left.\mathrm{CH}_{3}-27\right), 0.68\left(3 \mathrm{H}, \mathrm{CH}_{3}-28\right)$.

\section{3ß-O-Glutaryl-18- $\beta$-glycyrrhetinic acid (6)}

Positive FABMS $m / z$ 585.5.5 $(\mathrm{M}+\mathrm{H})^{+}$; HR-FABMS calcd for $\mathrm{C}_{35} \mathrm{H}_{51} \mathrm{O}_{7} 583.3635$, found 583.3622. ${ }^{1} \mathrm{H}$ NMR $\left(\mathrm{CDCl}_{3} / \mathrm{Py}-\mathrm{d}_{5}\right) \delta 5.81(1 \mathrm{H}, \mathrm{s}, \mathrm{H}-12), 4.61(1 \mathrm{H}, \mathrm{dd}, J=5.5 \mathrm{~Hz}, J=11.0$ $\mathrm{Hz}, \mathrm{H}-3), 2.91\left(1 \mathrm{H}, \mathrm{d}, J=13.5 \mathrm{~Hz}, \mathrm{H}_{2 \alpha}\right), 2.46-2.55\left(4 \mathrm{H}, \mathrm{m}, 2 \times \mathrm{COCH}_{2}\right), 2.01-2.12(2 \mathrm{H}, \mathrm{m}$, $\mathrm{CH}_{2}-3$ '), 1.37 (3H, s. $\left.\mathrm{CH}_{3}-29\right), 1.26\left(3 \mathrm{H}\right.$, s. $\left.\mathrm{CH}_{3}-25\right), 1.17$ (3H, s. $\left.\mathrm{CH}_{3}-23\right), 1.09$ (3H, s. $\left.\mathrm{CH}_{3}-24\right), 0.89$ (6H, s, $\mathrm{CH}_{3}-26$ and $\left.\mathrm{CH}_{3}-27\right), 0.80\left(3 \mathrm{H}, \mathrm{CH}_{3}-28\right)$.

\section{3 $\beta$-O-Diglycolyl-18- $\beta$-glycyrrhetinic acid (7)}

Positive FABMS $m / z 587.4(\mathrm{M}+\mathrm{H})^{+}$; HR-FABMS calcd for $\mathrm{C}_{34} \mathrm{H}_{49} \mathrm{O}_{8} 585.3427$, found 585.3433. ${ }^{1} \mathrm{H}$ NMR $\left(\mathrm{CDCl}_{3} / \mathrm{Py}-\mathrm{d}_{5}\right) \delta 5.65(1 \mathrm{H}, \mathrm{s}, \mathrm{H}-12), 4.54(1 \mathrm{H}, \mathrm{dd}, J=5.5 \mathrm{~Hz}, J=11.0$ $\mathrm{Hz}, \mathrm{H}-3), 4.19,4.18$ (each $2 \mathrm{H}$, each s, $\left.2 \times \mathrm{OCH}_{2}\right), 2.73\left(1 \mathrm{H}, \mathrm{d}, J=13.5 \mathrm{~Hz}, \mathrm{H}_{2 \alpha}\right), 1.27(3 \mathrm{H}, \mathrm{s}$. $\left.\mathrm{CH}_{3}-29\right), 1.12$ (3H, s. $\left.\mathrm{CH}_{3}-25\right), 1.04$ (3H, s. $\left.\mathrm{CH}_{3}-23\right), 1.00$ (3H, s. $\left.\mathrm{CH}_{3}-24\right), 0.78,0.76$ (each $3 \mathrm{H}$, each s, $\mathrm{CH}_{3}-26$ and $\left.\mathrm{CH}_{3}-27\right), 0.70\left(3 \mathrm{H}, \mathrm{CH}_{3}-28\right)$.

\section{3ß-O-Adipoyl-18- $\beta$-glycyrrhetinic acid (8)}

Negative FABMS $m / z 597.3(\mathrm{M}-\mathrm{H})^{-}$, HR-FABMS calcd for $\mathrm{C}_{36} \mathrm{H}_{53} \mathrm{O}_{7} 597.3791$, found 597.3801. ${ }^{1} \mathrm{H}$ NMR $\left(\mathrm{CDCl}_{3} / \mathrm{Py}-\mathrm{d}_{5}\right) \delta 5.71(1 \mathrm{H}, \mathrm{s}, \mathrm{H}-12), 4.50(1 \mathrm{H}, \mathrm{dd}, J=5.4 \mathrm{~Hz}, J=11.5$ $\mathrm{Hz}, \mathrm{H}-3), 2.78\left(1 \mathrm{H}, \mathrm{d}, J=13.5 \mathrm{~Hz}, \mathrm{H}_{2 \alpha}\right), 2.32-2.34\left(4 \mathrm{H}, \mathrm{m}, 2 \times \mathrm{COCH}_{2}\right), 1.36\left(3 \mathrm{H}, \mathrm{s} . \mathrm{CH}_{3}-29\right)$, 
1.20 (3H, s. $\left.\mathrm{CH}_{3}-25\right), 1.13$ (3H, s. $\left.\mathrm{CH}_{3}-23\right), 1.10$ (3H, s. $\left.\mathrm{CH}_{3}-24\right), 0.85$ (6H, s, $\mathrm{CH}_{3}-26$ and $\left.\mathrm{CH}_{3}-27\right), 0.79\left(3 \mathrm{H}, \mathrm{CH}_{3}-28\right)$.

\section{Glycyrrhetinic acid propionate (10)}

Negative FABMS $m / z 525.3(\mathrm{M}-\mathrm{H})^{-}$, HR-FABMS calcd for $\mathrm{C}_{33} \mathrm{H}_{49} \mathrm{O}_{5} 525.3580$, found 525.3586. ${ }^{1} \mathrm{H}$ NMR $\delta 5.70(1 \mathrm{H}, \mathrm{s}, \mathrm{H}-12), 4.52(1 \mathrm{H}, \mathrm{dd}, J=5.0 \mathrm{~Hz}, J=11.0 \mathrm{~Hz}, \mathrm{H}-3), 2.79$ $\left(1 \mathrm{H}, \mathrm{d}, J=13.5 \mathrm{~Hz}, \mathrm{H}_{2 \alpha}\right), 2.32\left(2 \mathrm{H}, \mathrm{t}, J=7.5 \mathrm{~Hz}, \mathrm{CH}_{2} \mathrm{CH}_{3}\right), 1.37$ (3H, s. $\left.\mathrm{CH}_{3}-29\right), 1.23(3 \mathrm{H}$, s. $\left.\mathrm{CH}_{3}-25\right), 1.17\left(3 \mathrm{H}\right.$, s. $\left.\mathrm{CH}_{3}-23\right), 1.13\left(3 \mathrm{H}\right.$, s. $\left.\mathrm{CH}_{3}-24\right), 1.13\left(3 \mathrm{H}, \mathrm{d}, J=7.3 \mathrm{~Hz}, \mathrm{CH}_{2} \mathrm{C}_{3}\right)$, 0.87, 0.88 (each $3 \mathrm{H}$, each s, $\mathrm{CH}_{3}-26$ and $\left.\mathrm{CH}_{3}-27\right), 0.83\left(3 \mathrm{H}, \mathrm{CH}_{3}-28\right)$.

\section{Glycyrrhetinic acid isobutytate (11)}

Negative FABMS $m / z 539.3(\mathrm{M}-\mathrm{H})^{-}$, HR-FABMS calcd for $\mathrm{C}_{34} \mathrm{H}_{51} \mathrm{O}_{5} 539.3765$, found 539.3745. ${ }^{1} \mathrm{H}$ NMR $\delta 5.71(1 \mathrm{H}, \mathrm{s}, \mathrm{H}-12), 4.50(1 \mathrm{H}, \mathrm{dd}, J=4.4 \mathrm{~Hz}, J=11.1 \mathrm{~Hz}, \mathrm{H}-3), 2.79$ $\left(1 \mathrm{H}, \mathrm{d}, J=13.6 \mathrm{~Hz}, \mathrm{H}_{2 \alpha}\right), 2.54\left(1 \mathrm{H}\right.$, qui, $\left.J=7.0 \mathrm{~Hz}, \mathrm{C} \underline{\mathrm{H}}-\left[\mathrm{CH}_{3}\right]_{2}\right), 1.37$ (3H, s. $\left.\mathrm{CH}_{3}-29\right), 1.23$ $\left(3 \mathrm{H}\right.$, s. $\left.\mathrm{CH}_{3}-25\right), 1.18,1.16$ (each $3 \mathrm{H}$, each d. $\left.J=6.7 \mathrm{~Hz},\left[\mathrm{CH}_{3}\right]_{2} \mathrm{CH}\right), 1.17\left(3 \mathrm{H}, \mathrm{s}, \mathrm{CH}_{3}-23\right)$, 1.13 (3H, s. $\left.\mathrm{CH}_{3}-24\right), 0.89,0.87$ (each $3 \mathrm{H}$, each s, $\mathrm{CH}_{3}-26$ and $\left.\mathrm{CH}_{3}-27\right), 0.84\left(3 \mathrm{H}, \mathrm{CH}_{3}-28\right)$.

\section{3 $\beta$-O-(trans- $\beta$-Hydromuconyl)-18- $\beta$-glycyrrhetinic acid (12)}

Negative FABMS $m / z 595.3(\mathrm{M}-\mathrm{H})^{-}$, HR-FABMS calcd for $\mathrm{C}_{36} \mathrm{H}_{51} \mathrm{O}_{7} 595.3635$, found 595.3649. ${ }^{1} \mathrm{H}$ NMR $\delta 5.72(1 \mathrm{H}, \mathrm{d}, J=5.0 \mathrm{~Hz}, \mathrm{C}=\mathrm{H}-3$ ') $5.69(2 \mathrm{H}, \mathrm{s}, \mathrm{C}=\mathrm{H}-2$ ' and $\mathrm{H}-12), 4.52$ $\left(1 \mathrm{H}, \mathrm{dd}, J=4.5 \mathrm{~Hz}, J=11.5 \mathrm{~Hz}, \mathrm{H}-3\right.$ ), $3.14,3.09$ (each $1 \mathrm{H}$, each d, $J=5.4 \mathrm{~Hz}, \mathrm{CH}_{2}-\mathrm{COO}$ ), $2.80\left(1 \mathrm{H}, \mathrm{d}, J=14.0 \mathrm{~Hz}, \mathrm{H}_{2 \alpha}\right), 1.37\left(3 \mathrm{H}\right.$, s. $\left.\mathrm{CH}_{3}-29\right), 1.22\left(3 \mathrm{H}, \mathrm{s} . \mathrm{CH}_{3}-25\right), 1.16(3 \mathrm{H}, \mathrm{s}$. $\mathrm{CH}_{3}-23$ ), 1.12 (3H, s. $\left.\mathrm{CH}_{3}-24\right), 0.87,0.86$ (each $3 \mathrm{H}$, each s, $\mathrm{CH}_{3}-26$ and $\left.\mathrm{CH}_{3}-27\right), 0.83$ (3H, $\left.\mathrm{CH}_{3}-28\right)$.

\section{3ß-O-benzoyl-18- $\beta$-glycyrrhetinic acid (13)}

Negative FABMS $m / z 573.3(\mathrm{M}-\mathrm{H})^{-}$, HR-FABMS calcd for $\mathrm{C}_{37} \mathrm{H}_{49} \mathrm{O}_{5} 597.3592$, found 573.3580. ${ }^{1} \mathrm{H}$ NMR $\delta 8.04(2 \mathrm{H}, \mathrm{d}, J=7.0 \mathrm{~Hz}, 2 \times \mathrm{H}-o-\mathrm{Ar}), 7.55(1 \mathrm{H}, \mathrm{t}, J=7.2 \mathrm{~Hz}, \mathrm{H}-p-\mathrm{Ar})$, $7.44(2 \mathrm{H}, \mathrm{t}, J=7.2 \mathrm{~Hz}, 2 \times \mathrm{H}-m-\mathrm{Ar}), 5.73(1 \mathrm{H}, \mathrm{s}, \mathrm{H}-12), 4.76(1 \mathrm{H}, \mathrm{dd}, J=5.0 \mathrm{~Hz}, J=10.8 \mathrm{~Hz}$, $\mathrm{H}-3), 2.85\left(1 \mathrm{H}, \mathrm{d}, J=13.5 \mathrm{~Hz}, \mathrm{H}_{2 \alpha}\right), 1.40\left(3 \mathrm{H}, \mathrm{s} . \mathrm{CH}_{3}-29\right), 1.23\left(3 \mathrm{H}, \mathrm{s} . \mathrm{CH}_{3}-25\right), 1.22(3 \mathrm{H}, \mathrm{s}$. $\left.\mathrm{CH}_{3}-23\right), 1.15$ (3H, s. $\left.\mathrm{CH}_{3}-24\right), 1.04\left(3 \mathrm{H}, \mathrm{s}, \mathrm{CH}_{3}-26\right), 0.95\left(3 \mathrm{H}, \mathrm{s}, \mathrm{CH}_{3}-27\right), 0.85(3 \mathrm{H}$, $\left.\mathrm{CH}_{3}-28\right)$.

\section{Glycyrrhetinic acid-4-acetylphenoxyl acetate (14)}

Positive FABMS $m / z 661.4(\mathrm{M}+\mathrm{H})^{+}$, HR-FABMS calcd for $\mathrm{C}_{41} \mathrm{H}_{57} \mathrm{O}_{7} 661.4104$, found 661.4097. ${ }^{1} \mathrm{H}$ NMR $\delta$ 7.92, 6.92, (each $2 \mathrm{H}$, each d, $\left.J=9.0 \mathrm{~Hz}, 4 \times \mathrm{H}-\mathrm{Ar}\right), 5.68(1 \mathrm{H}, \mathrm{s}, \mathrm{H}-12)$, $4.63(1 \mathrm{H}, \mathrm{dd}, J=5.5 \mathrm{~Hz}, J=11.0 \mathrm{~Hz}, \mathrm{H}-3), 2.79\left(1 \mathrm{H}, \mathrm{d}, J=14.0 \mathrm{~Hz}, \mathrm{H}_{2 \alpha}\right), 2.54(3 \mathrm{H}, \mathrm{s}$, $\left.\mathrm{COCH}_{3}\right), 1.34\left(3 \mathrm{H}\right.$, s. $\left.\mathrm{CH}_{3}-29\right), 1.20\left(3 \mathrm{H}\right.$, s. $\left.\mathrm{CH}_{3}-25\right), 1.12\left(3 \mathrm{H}\right.$, s. $\left.\mathrm{CH}_{3}-23\right), 1.10$ (3H, s. $\left.\mathrm{CH}_{3}-24\right), 0.80\left(6 \mathrm{H}, \mathrm{s}, \mathrm{CH}_{3}-26\right.$ and $\left.\mathrm{CH}_{3}-27\right), 0.78\left(3 \mathrm{H}, \mathrm{CH}_{3}-28\right)$.

\section{Glycyrrhetinic acid-m-phenylene monoacetate (15)}

Positive FABMS $m / z 647.5(\mathrm{M}+\mathrm{H})^{+}$; HR-FABMS calcd for $\mathrm{C}_{40} \mathrm{H}_{53} \mathrm{O}_{7} 645.3791$, found 645.3771. ${ }^{1} \mathrm{H}$ NMR $\delta 7.18-7.35(5 \mathrm{H}, \mathrm{m}, 5 \times \mathrm{H}-\mathrm{Ar}), 5.67(1 \mathrm{H}, \mathrm{s}, \mathrm{H}-12), 4.49(1 \mathrm{H}, \mathrm{d}, J=4.2 \mathrm{~Hz}$, $\mathrm{H}-3), 3.63\left(4 \mathrm{H}, \mathrm{d}, J=11.0 \mathrm{~Hz}, 2 \times \mathrm{CH}_{2}-\Phi\right), 2.76\left(1 \mathrm{H}, \mathrm{d}, J=14.5 \mathrm{~Hz}, \mathrm{H}_{2 \alpha}\right), 1.35(3 \mathrm{H}, \mathrm{s}$. $\left.\mathrm{CH}_{3}-29\right), 1.20$ (3H, s. $\left.\mathrm{CH}_{3}-25\right), 1.13$ (3H, s. $\left.\mathrm{CH}_{3}-23\right), 1.11$ (3H, s. $\left.\mathrm{CH}_{3}-24\right), 0.81,0.79$ (each $3 \mathrm{H}$, each s, $\mathrm{CH}_{3}-26$ and $\left.\mathrm{CH}_{3}-27\right), 0.76\left(3 \mathrm{H}, \mathrm{CH}_{3}-28\right)$.

\section{Glycyrrhetinic acid-p-phenylene monoacetate (16)}

Negative FABMS $m / z 645.3(\mathrm{M}-\mathrm{H})^{-}$, HR-FABMS calcd for $\mathrm{C}_{40} \mathrm{H}_{53} \mathrm{O}_{7} 645.3814$, found 645.3791. ${ }^{1} \mathrm{H}$ NMR $\delta 7.26$ (4H, s, 4×H-Ar), $5.68(1 \mathrm{H}, \mathrm{s}, \mathrm{H}-12), 4.53$ (1H, m, H-3), 3.63 (4H, 
d, $\left.J=15.5 \mathrm{~Hz}, 2 \times \mathrm{CH}_{2}-\Phi\right), 2.78\left(1 \mathrm{H}, \mathrm{d}, J=13.0 \mathrm{~Hz}, \mathrm{H}_{2 \alpha}\right), 1.36\left(3 \mathrm{H}, \mathrm{s} . \mathrm{CH}_{3}-29\right), 1.21(3 \mathrm{H}, \mathrm{s}$. $\left.\mathrm{CH}_{3}-25\right), 1.15$ (3H, s. $\left.\mathrm{CH}_{3}-23\right), 1.12$ (3H, s. $\left.\mathrm{CH}_{3}-24\right), 0.82\left(6 \mathrm{H}, \mathrm{s}, \mathrm{CH}_{3}-26\right.$ and $\left.\mathrm{CH}_{3}-27\right), 0.79$ $\left(3 \mathrm{H}, \mathrm{CH}_{3}-28\right)$.

\section{Glycyrrhetinic acid monoisophthalate (17)}

Negative FABMS $m / z 617.3(\mathrm{M}-\mathrm{H})^{-}$, HR-FABMS calcd for $\mathrm{C}_{38} \mathrm{H}_{49} \mathrm{O}_{7} 617.3478$, found 617.3483. ${ }^{1} \mathrm{H}$ NMR $\left(\mathrm{CDCl}_{3} / \mathrm{Py} \mathrm{d}_{5}\right) \delta 8.76(1 \mathrm{H}, \mathrm{s}, \mathrm{H}-2$ '-Ar), 8.28, 8.18 (each $1 \mathrm{H}$, each d, $J=$ $7.8 \mathrm{~Hz}, 2 \times \mathrm{H}-\mathrm{Ar}), 7.50(1 \mathrm{H}, \mathrm{t}, J=7.8 \mathrm{~Hz}, \mathrm{H}-5$ '-Ar), $5.73(1 \mathrm{H}, \mathrm{s}, \mathrm{H}-12), 4.78(1 \mathrm{H}, \mathrm{dd}, \mathrm{J}=4.8$ $\mathrm{Hz}, \mathrm{J}=11.0 \mathrm{~Hz}, \mathrm{H}-3), 2.85\left(1 \mathrm{H}, \mathrm{d}, J=14.0 \mathrm{~Hz}, \mathrm{H}_{2 \alpha}\right), 1.38\left(3 \mathrm{H}, \mathrm{s} . \mathrm{CH}_{3}-29\right), 1.20(3 \mathrm{H}, \mathrm{s}$. $\left.\mathrm{CH}_{3}-25\right), 1.19$ (3H, s. $\left.\mathrm{CH}_{3}-23\right), 1.12$ (3H, s. $\left.\mathrm{CH}_{3}-24\right), 1.04\left(3 \mathrm{H}, \mathrm{s}, \mathrm{CH}_{3}-26\right), 0.93$ (3H, s, $\left.\mathrm{CH}_{3}-27\right), 0.80\left(3 \mathrm{H}, \mathrm{CH}_{3}-28\right)$.

\section{Glycyrrhetinic acid monoterephthalate (18)}

Negative FABMS $m / z 617.3(\mathrm{M}-\mathrm{H})^{-}$, HR-FABMS calcd for $\mathrm{C}_{36} \mathrm{H}_{53} \mathrm{O}_{7} 617.3478$, found 617.3464. ${ }^{1} \mathrm{H}$ NMR $\left(\mathrm{CDCl}_{3} / \mathrm{Py}_{-} \mathrm{d}_{5}\right) \delta 8.14,8.05$ (each $2 \mathrm{H}$, each d, $\left.J=8.5 \mathrm{~Hz}, 4 \times \mathrm{H}-\mathrm{Ar}\right), 5.71$ $(1 \mathrm{H}, \mathrm{s}, \mathrm{H}-12), 4.74(1 \mathrm{H}, \mathrm{dd}, J=4.5 \mathrm{~Hz}, J=10.0 \mathrm{~Hz}, \mathrm{H}-3), 2.83\left(1 \mathrm{H}, \mathrm{d}, J=14.0 \mathrm{~Hz}, \mathrm{H}_{2 \alpha}\right), 1.35$ (3H, s. $\left.\mathrm{CH}_{3}-29\right), 1.19$ (3H, s. $\left.\mathrm{CH}_{3}-25\right), 1.16\left(3 \mathrm{H}\right.$, s. $\left.\mathrm{CH}_{3}-23\right), 1.09$ (3H, s. $\left.\mathrm{CH}_{3}-24\right), 0.99$ (3H, s, $\left.\mathrm{CH}_{3}-26\right), 0.91\left(3 \mathrm{H}, \mathrm{s}, \mathrm{CH}_{3}-27\right), 0.78\left(3 \mathrm{H}, \mathrm{CH}_{3}-28\right)$.

\section{HPLC purification of the synthesized GLA derivatives}

\section{Proteasome assay}

Proteasome assay kits were purchased from Calbiochem, San Diego, CA. The effect of GLA and its analogs on the 20S proteasome activity was assayed following the protocol provided by the manufacturer. The major components of the assay mixture are human $20 \mathrm{~S}$ proteasomes, fluorogenic peptide substrates and the proteasome activator PA28. The assay was designed to measure hydrolysis of the fluorogenic substrates Suc-Leu-Leu-Val-Tyr-AMC, (Z)-LLE-bNA, and Bz-VGR-AMC in the presence of the proteasome activator PA28. Suc-Leu-Leu-Val-Tyr$\mathrm{AMC}$ is frequently used to detect the chymotrypsin-like activity of 20S proteasomes. The trypsin-like and caspase-like activities of the 20S proteasome were determined using the fluorogenic substrates Bz-VGR-AMC and (Z)-LLE-bNA, respectively. Fluorescence generated from the proteolytic reaction in the presence of various concentrations of GLA or its analogs was measured using a Bio-Tek fluorometer (Winooski, Vermont).

For proteasome inhibition, various concentrations of GLA derivatives were tested in the presence of $16 \mu \mathrm{g} / \mathrm{ml}$ of PA28. The known proteasome inhibitors LLM-F (Boston Biochem, Cambridge, MA, USA) and lactacystin (Sigma-Aldrich, St. Louis, MO) were used as controls for the proteasome inhibition assays. The 50\% inhibitory concentration (IC50) is defined as the inhibitory concentration that reduces the reaction rate by $50 \%$. The velocity of reaction, $\triangle \mathrm{RFU}(360 / 460 \mathrm{~nm}) /$ minute, was plotted against the log-concentration of the inhibitor to determine the IC50.

\section{Cell-based proteasome assay}

To determine the effect of GLA derivatives on proteasomes in cells, a Promega cell-based assay was used in this study. MT4 cells (4,000 cells) were treated with GLA derivatives or known proteasome inhibitors in serum free medium at $37^{\circ} \mathrm{C}$ for 3 hours. MT4 cells are human $\mathrm{T}$ cells isolated from a patient with adult T-cell leukemia. MT4 cells were obtained from the NIH AIDS Research and Reference Reagent Program. The drug-treated MT4 cells were incubated with the Promega Proteasome-Glo Cell-Based Assay Reagent (Promega Bioscience, Madison, WI) for 10 minutes. The chymotrypsin-like proteasome activity was detected as the relative light unit (RLU) generated from the cleaved substrate in the reagent. Luminescence 
generated from each reaction condition was detected with a PerkinElmer Victor-3 luminometer (Shelton, CT, USA).

\section{Acknowledgements}

The authors wish to thank Shannon Meroney-Davis for her help on editing this manuscript. The authors are grateful to Dr. George Dubay of the Department of Chemistry and Dr. Anthony Ribeiro of the NMR Spectroscopy Center of Duke University for their help and assistance on mass and NMR spectroscopy data collection.. This work was supported in part by grant from Centers for AIDS Research at Duke University (LH), the National Institutes of Health Grants AI65310 (CHC), and CA17625 and AI33066 (KHL).

\section{References}

1. Dalton L. C\&EN 2002;80:37.

2. Davis E, Morris D. Mol. Cell. Endo 1991;78:1-6.

3. Monder C, Stewart PM, Lakshmi V, Valentino R, Burt D, Edwards CRW. Endocrinol 1989;125:10461053.

4. Buhler H, Perschel FH, Hierholzer K. Biochim. Biophys. Acta 1991;1075:206-212. [PubMed: 1954223]

5. Salvi M, Fiore C, Armanini D, Toninello A. Biochem. Pharmacol 2003;66:2375-2379. [PubMed: 14637195]

6. Ciechanover A. Nat. Rev. Mol. Cell Biol 2005;6:79-86. [PubMed: 15688069]

7. Hershko A, Ciechanover A. Annu. Rev. Biochem 1998;67:425-479. [PubMed: 9759494]

8. Nalepa G, Rolfe M, Harper JW. Nat. Rev. Drug Discov 2006;5:596-613. [PubMed: 16816840]

9. Rechsteiner M, Hill CP. Trends Cell Biol 2005;15:27-33. [PubMed: 15653075]

10. Groll M, Heinemeyer W, Jager S, Ullrich T, Bochtler M, Wolf DH, Huber R. PNAS 1999;96:1097610983. [PubMed: 10500111]

11. Kane RC, Bross PF, Farrell AT, Pazdur R. Oncologist 2003;8:508-513. [PubMed: 14657528]

12. Kisselev AF, Callard A, Goldberg AL. J. Biol. Chem 2006;281:8582-8590. [PubMed: 16455650]

13. Huang L, Ho P, Chen C-H. FEBS Letters 2007;581:4955-4959. [PubMed: 17904555]

14. Heinemeyer W, Kleinschmidt JA, Saidowsky J, Escher C, Wolf DH. EMBO J 1991;10:555-562. [PubMed: 2001673]

15. Gueckel R, Enenkel C, Wolf DH, Hilt W. J. Biol. Chem 1998;273:19443-19452. [PubMed: 9677364]

16. Richter-Ruoff B, Wolf DH, Hochstrasser M. FEBS Lett 1994;354:50-52. [PubMed: 7957900] Erratum in: FEBS Lett. 1995, 358, 104.

17. Seufert W, Jentsch S. EMBO J 1992;11:3077-3080. [PubMed: 1322295]

18. Yu D, Sakurai Y, Chen CH, Chang FR, Huang L, Kashiwada Y, Lee KH. J. Med. Chem 2006;49:54625469. [PubMed: 16942019]

19. Goldberg AL. Biochem. Soc. Trans 2007;35(Pt 1):12-17. [PubMed: 17212580]

20. Zhang J, Cheng Z-H, Yu B-Y, Cordell GA, Qiu SX. Tetrahedron Lett 2005;46:2337-2340.

21. Dick LR, Cruikshank AA, Grenier L, Melandri FD, Nunes SL, Stein RL. J. Biol. Chem 1996;271:7273-7276. [PubMed: 8631740]

22. Sato H, Goto W, Yamamura J, Kurokawa M, Kageyama S, Takahara T, Watanabe A, Shiraki K. Antiviral Res 1996;30:171-177. [PubMed: 8783808]

23. Yamamura Y, Kawakami J, Santa T, Kotaki H, Uchino K, Sawada Y, Tanaka N, Iga T. J. Pharm. Sci 1992;81:1042-1046. [PubMed: 1432618] 


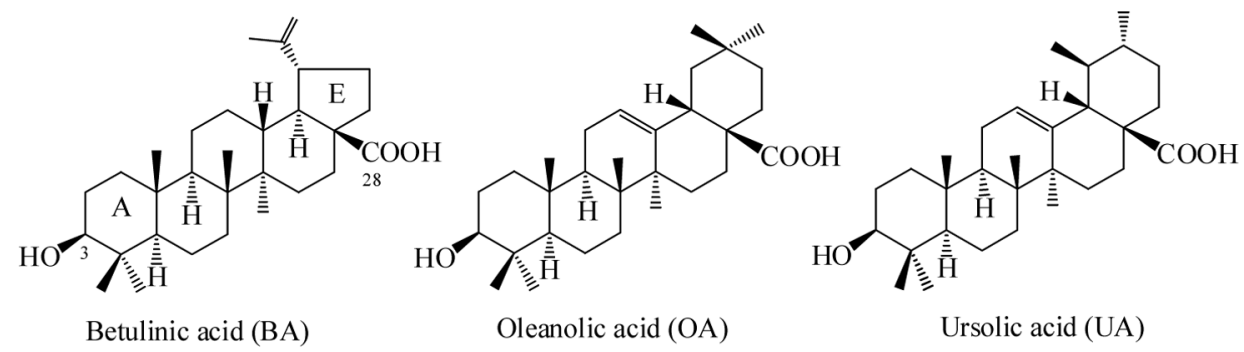

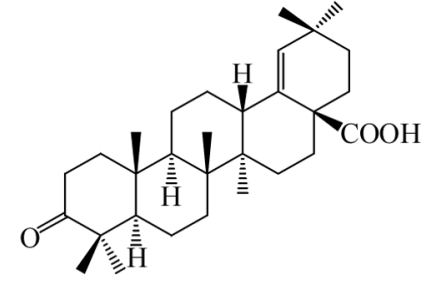

Moronic acid (MA)

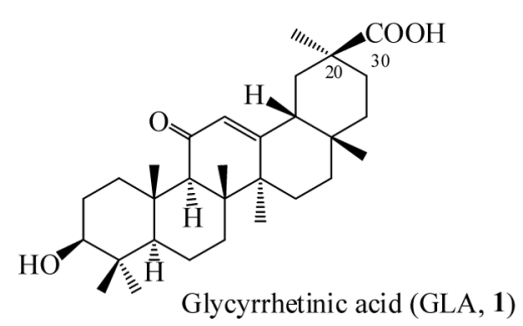

Figure 1. Chemical structure of triterpenes 


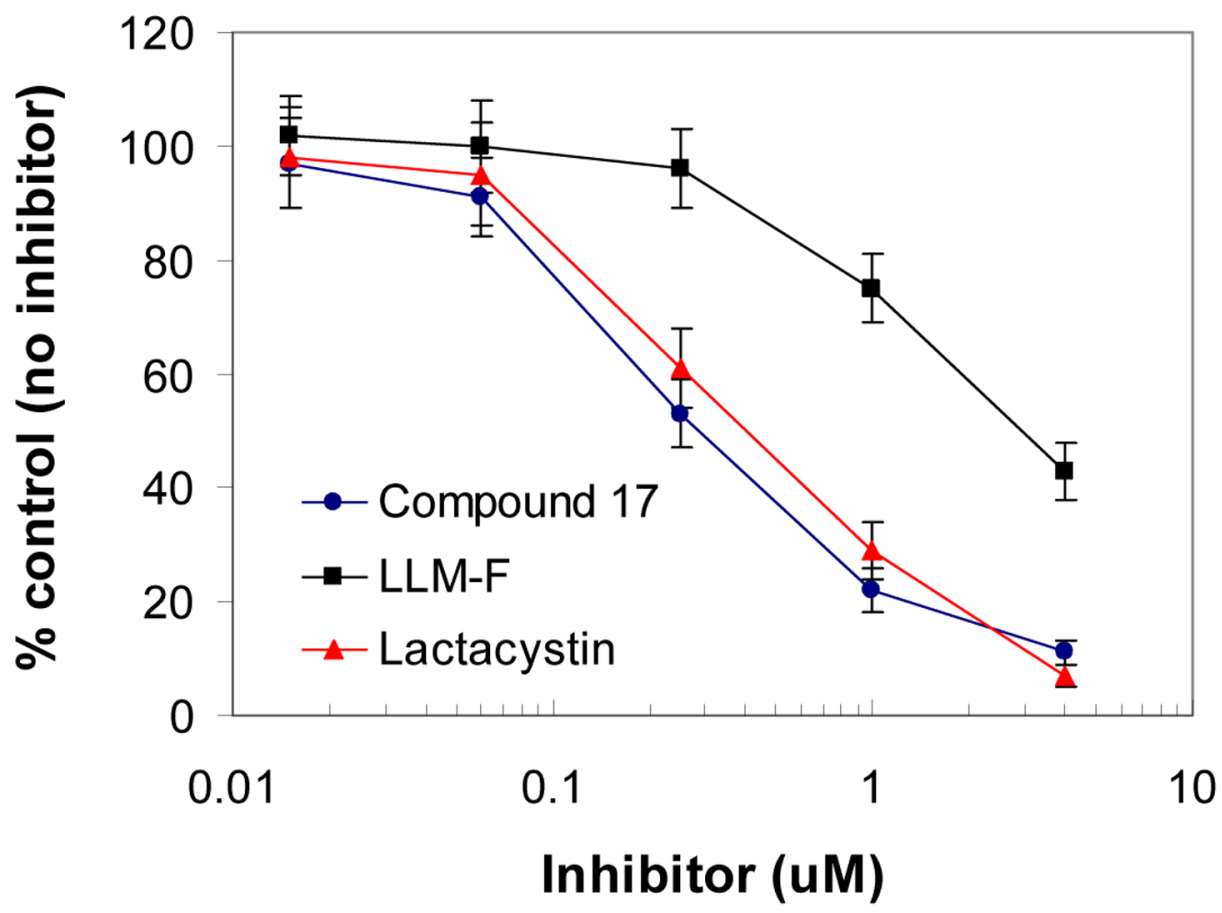

Figure 2. The GLA derivative 17 inhibited the chymotrypsin-like activity of the proteasome in MT4 cells

A Promega cell-based proteasome assay was used to determine the effect of compound $\mathbf{1 7}$ on the ChT-L activity of 20S proteasome (13). The known proteasome inhibitors LLM-f and lactacystin were used as positive controls for proteasome inhibition. MT4 cells are human T cells isolated from a patient with adult T-cell leukemia. The ChT-L proteasome activity was detected as the relative light unit (RLU) generated from the cleaved substrate in the assay. The $\%$ control is derived using the formula: $100 \times(\mathrm{RLU}$ with test compound) / (RLU from the control without compound). 

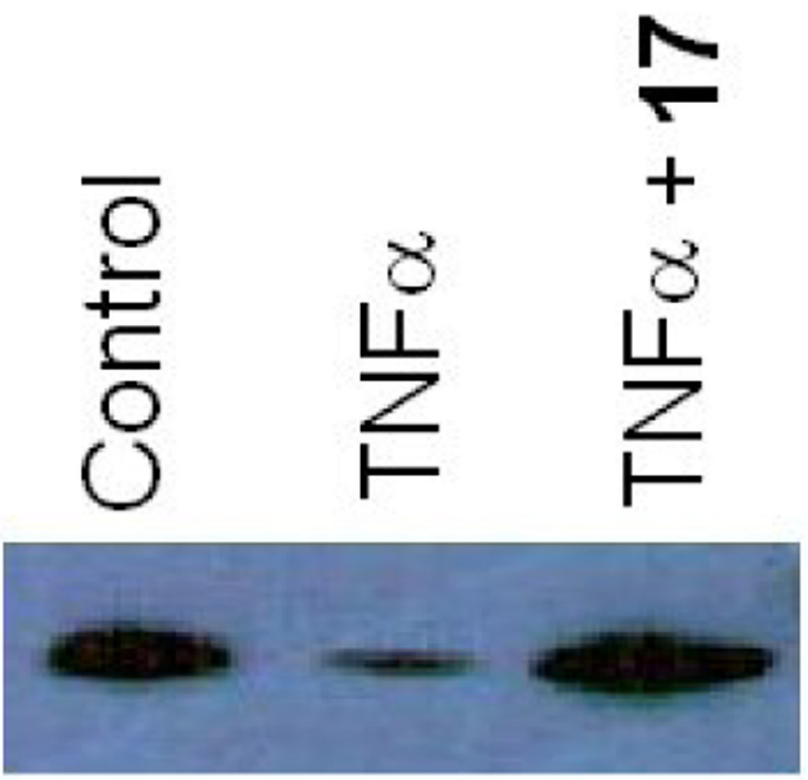

$\mathrm{I} \kappa \mathrm{B} \alpha$

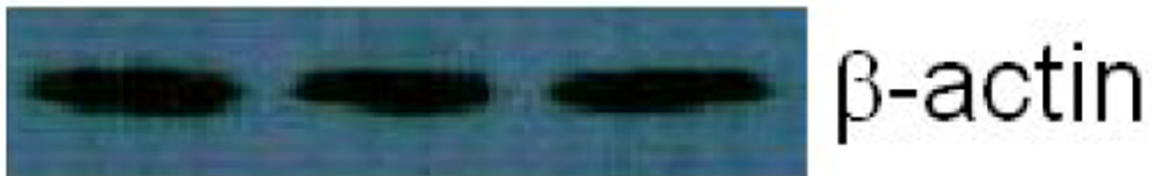

Figure 3.

The GLA derivative 17 inhibited degradation of IкB in MT4 cells. 
Table 1

${ }^{13} \mathrm{C}$ NMR spectral data (assignment) of compounds $\mathbf{8}, \mathbf{1 2}, \mathbf{1 7}$ and $\mathbf{1 8}^{a}$.

8

80.5 (C-3)

55.1 (C-5)

$62.0(\mathrm{C}-9)$

$199.6(\mathrm{C}-11)$

128.7 (C-12)

$170.0(\mathrm{C}-13)$

$179.3(\mathrm{C}-30)$

48.8

45.6

44.2

43.6

41.8

39.1
38.5

38.4

37.5

32.8

32.2

28.82

28.78

28.3

26.9

26.7

24.1

23.6

18.9

17.7
17.2

16.8

$\mathrm{C}$ on $\mathrm{C}-3$ side chain

$175.8(\mathrm{C}=\mathrm{O})$

$173.2(\mathrm{C}=\mathrm{O})$

$34.7\left(\mathrm{CH}_{2} \mathrm{CO}\right)$

$34.6\left(\mathrm{CH}_{2} \mathrm{CO}\right)$

$25.24\left(\mathrm{CH}_{2} \mathrm{CH}_{2} \mathrm{CO}\right)$

$25.21\left(\mathrm{CH}_{2} \mathrm{CH}_{2} \mathrm{CO}\right)$
12

$80.9(\mathrm{C}-3)$

$55.1(\mathrm{C}-5)$

$62.0(\mathrm{C}-9)$

$199.6(\mathrm{C}-11)$

128.7 (C-12)

$170.0(\mathrm{C}-13)$

$179.3(\mathrm{C}-30)$

48.8

45.6

44.2

43.6

41.8

39.1

38.8

38.5

37.5

32.8

32.3

31.7

28.83

28.78

28.3

26.9

26.7

24.1

23.6

18.8

17.7

17.1

16.8

$174.0(\mathrm{C}=\mathrm{O})$

$171.5(\mathrm{C}=\mathrm{O})$

$127.8(\mathrm{CH}=)$

$126.1(\mathrm{CH}=)$

$38.6\left(=\mathrm{CHCH}_{2}\right)$

$38.5\left(=\mathrm{CHC}_{2}{ }_{2}\right)$
17

$81.9(\mathrm{C}-3)$

55.1 (C-5)

62.0 (C-9)

$199.6(\mathrm{C}-11)$

128.7 (C-12)

170.1 (C-13)

179.3 (C-30)

48.8

45.6

44.2

43.6

41.8

39.1

38.8

38.5

37.5

32.8

32.3

28.83

28.80

28.4

26.9

26.7

24.1

23.7

18.9

17.7

17.3

16.8

$168.5(\mathrm{C}=\mathrm{O})$

$165.9(\mathrm{C}=\mathrm{O})$

134.5 (Ar-C)

133.6 (Ar-C)

133.4 (Ar-C)

131.9 (Ar-C)

131.3 (Ar-C)

129.3 (Ar-C)
18

$81.6(\mathrm{C}-3)$

55.1 (C-5)

62.0 (C-9)

$199.6(\mathrm{C}-11)$

128.7 (C-12)

$170.2(\mathrm{C}-13)$

179.5 (C-30)

48.8

45.7

44.3

43.6

-

39.1

38.7

38.5

37.5

32.8

32.3

31.7

28.9

28.8

28.4

26.9

26.7

24.1

23.7

18.9

17.7

17.3

16.8

$166.2^{b}(\mathrm{C}=\mathrm{O})$

$130.5^{c}(\mathrm{Ar}-\mathrm{C})$

$129.7^{d}(\mathrm{Ar}-\mathrm{C})$

${ }^{a}$ Recorded in pyridine- $\mathrm{d} 5$ at $125 \mathrm{MHz}$.

$b, c, d$ Overlapping signals. 


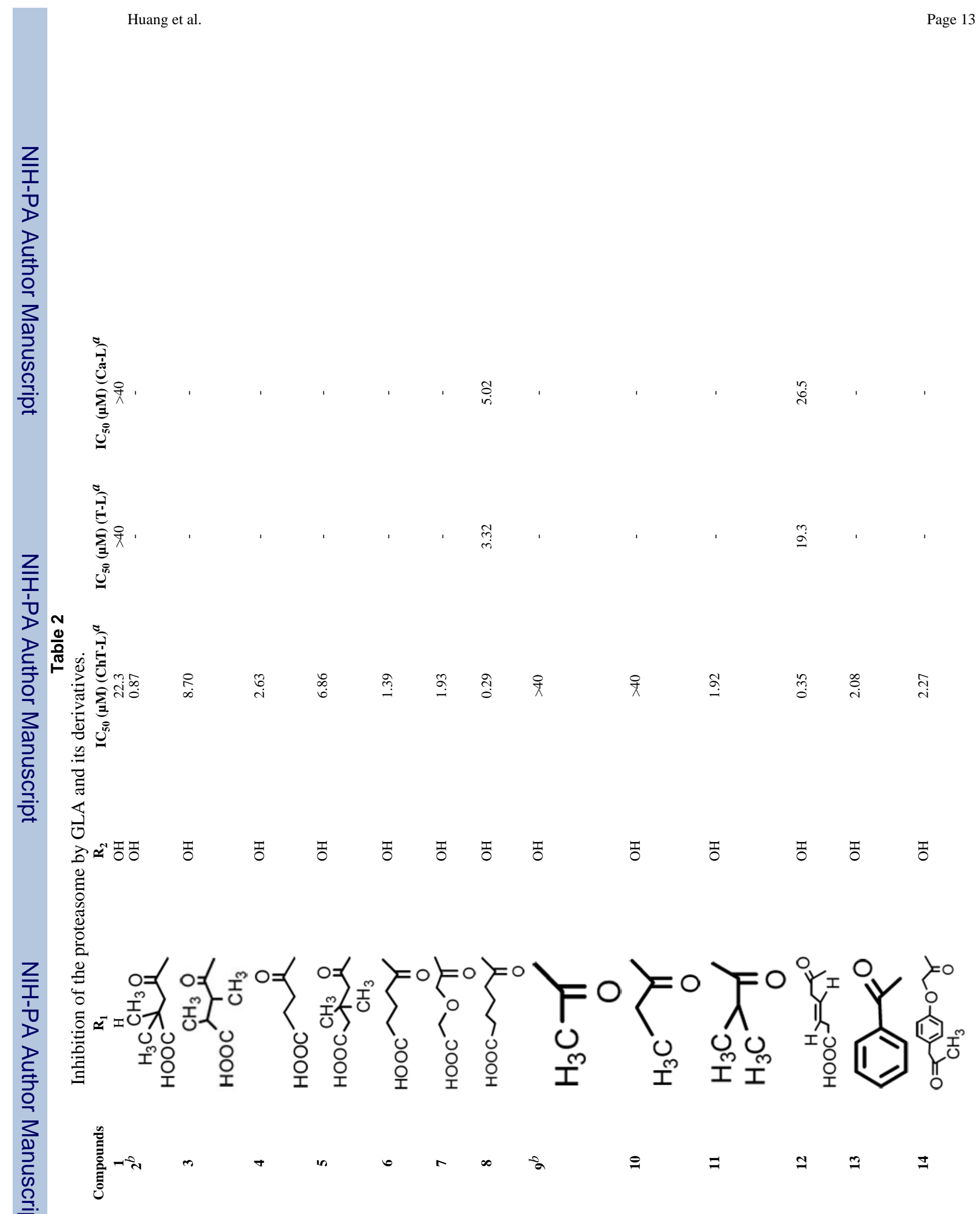

Bioorg Med Chem. Author manuscript; available in PMC 2009 July 15. 


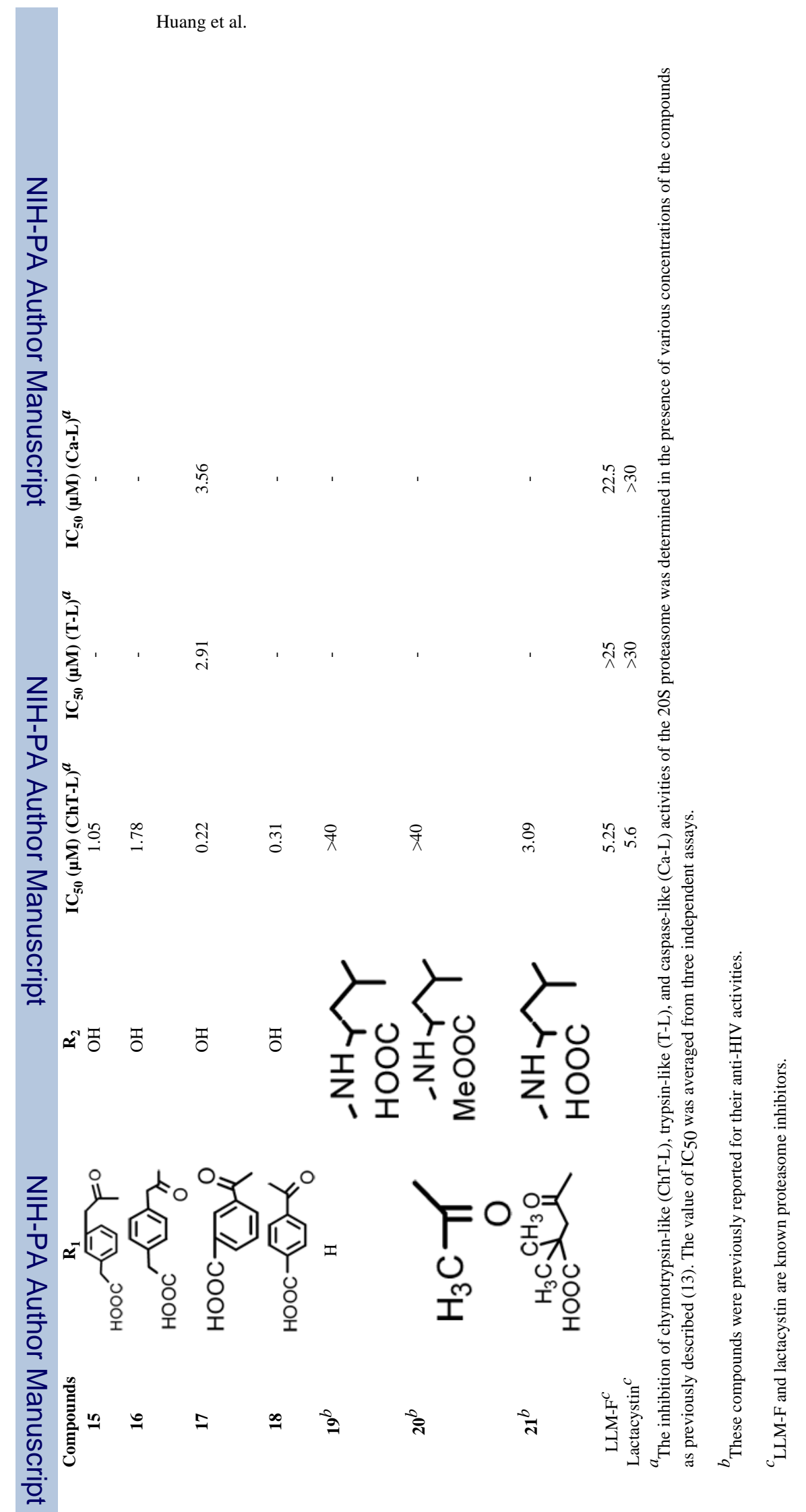

Bioorg Med Chem. Author manuscript; available in PMC 2009 July 15. 


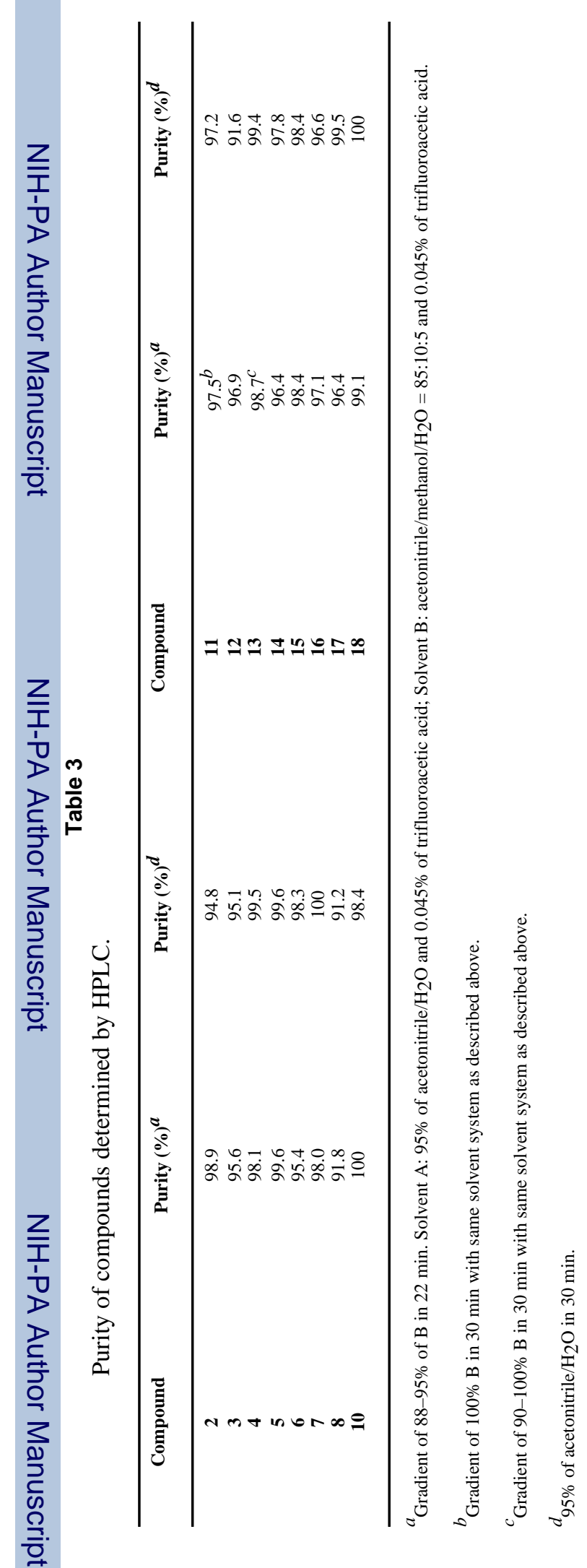

Bioorg Med Chem. Author manuscript; available in PMC 2009 July 15. 